\title{
Sinusoidal Signal Generation for Mixed-Signal BIST Using a Harmonic-Cancellation Technique
}

\author{
Manuel J. Barragan, Gildas Leger, Diego Vazquez, and Adoracion Rueda \\ Instituto de Microlectrónica de Sevilla, Centro Nacional de Microelectrónica \\ Consejo Superior de Investigaciones Científicas (CSIC) and Universidad de Sevilla \\ Av. Américo Vespucio s/n, 41092 Sevilla, Spain. \\ e-mail: manueljeimse-cnm.csic.es
}

\begin{abstract}
This work presents a technique for the generation of analog sinusoidal signals with high spectral quality and reduced circuitry resources. The proposed generation technique is based on a modified analog filter, that provides a sinusoidal output as the response to a DC input, combined with a harmonic cancellation technique. It has the attributes of digital programming and control, low area overhead, and low design effort, which make this approach very suitable as test stimulus generator for builtin test applications. Simulation results are provided in order to validate the proposed generation technique.
\end{abstract}

\section{INTRODUCTION}

The continuous advance in integration capabilities in current CMOS technologies enables the production of complex mixedsignal ICs. Despite this increase in complexity, design and manufacturing costs have remained reasonably contained due to steady developments in design tools and technological advances. However, in this evolution there is a simultaneous increase in the cost of testing and diagnosing these devices. Test is becoming a dominant factor in overall production cost [1].

The generation of spectrally pure sinusoidal signals has a wide variety of potential applications in the field of analog and mixed-signal testing, and represents a key point for many testing schemes. In fact, most of the analog and mixed-signal subsystems in a SoC, such as analog filters, A/D converters, signal conditioners, etc, can be characterized by applying a sinusoidal test stimulus and analyzing the response. Providing an efficient method for the on-chip generation of analog sinusoidal stimuli can be identified as a key point to extend many testing procedures to a full-BIST scheme.

This work proposes an efficient technique for the onchip generation of sinusoidal analog test stimuli with a high spectral purity. The proposed generation technique is based in the modified filtering technique previously proposed by the authors in [2], combined with a simple harmonic cancellation technique.

This paper is organized as follows. Section II reviews briefly some previous strategies for sine-wave generation. Section III presents the theoretical basis of the proposed generation technique, and then Section IV discusses its practical implementation. Section V provides some simulation results for validation. Finally, Section VI summarizes the main contributions of this work.

\section{PREvious WORKS}

Analog test stimulus generation for BIST applications continues to be a hot topic in the test research community. A number of different strategies for analog signal generation have been published for the last years [2]-[8].

A classical solution for the generation of sinusoidal signals is the closed-loop oscillator, that consists of a filtering section with a non-linear feedback mechanism [5], [6]. The quality of the generated signal depends on the linearity and selectivity of the filter and the shape of the non-linear function. Highly selective filters and smooth non-linear functions are needed for the generation of high-accuracy, low-distorted waveforms.

On the other hand, many of the proposed strategies for onchip generation of test signals adopt an open-loop scheme. These waveform generators are usually based on a digital pattern generator followed by a D/A conversion [2]-[4]. The digital pattern generator outputs a digital sequence, the D/A converter translates the digital pattern to the analog domain, and finally the signal is fed to an analog filter that attenuates any non-desired components in the output signal.

Some recent proposals for signal generation [7], [8] combine a closed-loop oscillator and a harmonic cancellation technique to provide very low THD sinusoidal signals. Our proposed generation technique makes use of a similar harmonic cancellation strategy, but instead of an oscillator, we propose the use of the efficient methodology for sine-wave generation presented by the authors in [2]. This generation strategy is based on a low-order linear system with programmable input elements, and offers the advantages of design simplicity and full digital control.

\section{THEORETICAL BASIS}

Fig. 1 shows a conceptual block diagram of a signal generator with harmonic cancellation. It is composed of a set of $n$ matched sinusoidal signal generators that provide time-delayed versions of the same sinusoidal signal. The timedelayed signals are added to provide harmonic cancellation on the output sine-wave signal. Let us discuss each stage separately.

\section{A. Sine-wave generation technique}

In this paper we propose the application of harmonic cancellation to the sine-wave generation technique presented 


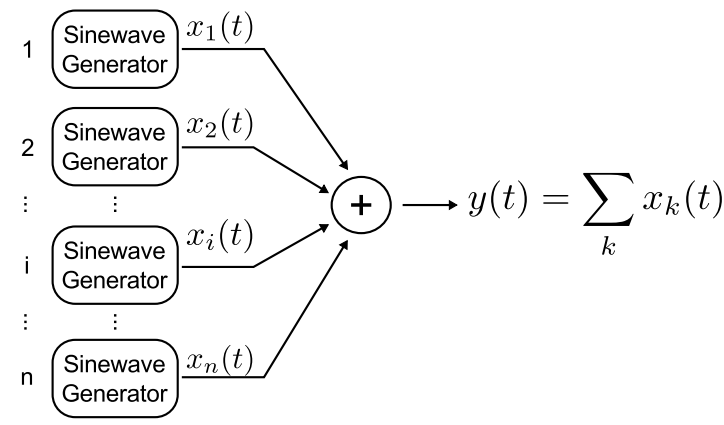

Fig. 1. Conceptual block diagram of the proposed sinusoidal generator with harmonic cancellation. Each $x_{i}(t)$ is a time-delayed version of the same sinewave.
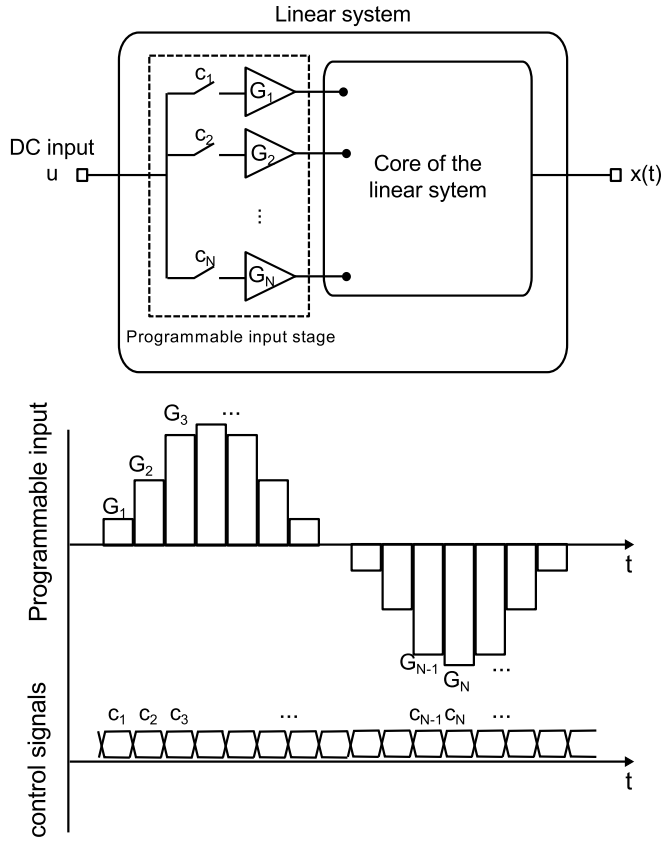

Fig. 2. Block diagram of our basic sinusoidal signal generator.

in [2]. Fig. 2 shows a conceptual block diagram of the selected generation strategy. The signal generator is a linear system whose input stage has been modified to provide a programmable gain. The generation strategy is based on weighting the programmable input levels as the values of a sampled sinusoid. This way, exciting the system by a DC level, $u$, and connecting the programmable input levels sequentially to the signal path, the input stage provides a sinusoidal stimulus to the core of the linear system. It can be proved that the output, $x(t)$, of this sinusoidal generator is given by [2],

$$
x(t)=u\{h(t) \otimes f(t)\}
$$

where $h(t)$ is the impulse response of the linear system, and $f(t)$ is the step-wise sine-wave defined by the programmable input levels. That is, the output of the linear system is a filtered and scaled version of the step-wise sine-wave $f(t)$. Selecting properly the impulse response $h(t)$ and the number of programmable levels in $f(t)$, it has been proved that this strategy can be used to implement efficient and precise sinusoidal generators [2].

The main drawback of this generator is that the linearity of the generated signal depends strongly on the number and accuracy of the programmable input levels. A high number of steps and precise step values are required for high linearity signal generation. However, in a practical implementation the number of programmable levels should remain low for a feasible design, which in turn increases the sensitivity to step errors due to mismatch between the programmable input elements, process or temperature variations, etc. This work will show that the application of harmonic cancellation techniques improves the linearity of the generated signal while reducing the sensitivity to input step errors.

\section{B. Harmonic cancellation}

Harmonic cancellation is a classical linearization method based on combining time-delayed versions of a periodic signal. This method achieves the suppression of unwanted harmonic components in the signal under consideration. Our proposed sine-wave generator takes advantage of this strategy for improving the spectral purity of the generated sinusoidal signal above the performance of the individual matched generators in Fig. 1.

Let us consider signal $x_{1}(t)$ generated by the first of the matched basic generators in Fig. 1. Signal $x_{1}(t)$ is a periodic signal that can be expressed as a Fourier series expansion,

$$
x_{1}(t)=\sum_{k=1}^{\infty} A_{k} \cos \left(k \omega_{0} t+\varphi_{k}\right)
$$

where $A_{k}$ and $\varphi_{k}$ are the amplitude and phase of harmonic component $k$ in signal $x_{1}(t)$, respectively, and $\omega_{0}$ is the fundamental frequency of $x_{1}(t)$.

Let us consider a time-shifted version of signal (2),

$$
\begin{aligned}
x_{1}(t+\Delta t)= & \sum_{k=1}^{\infty} A_{k} \cos (k \phi) \cos \left(k \omega_{0} t+\varphi_{k}\right) \\
& -\sum_{k=1}^{\infty} A_{k} \sin (k \phi) \sin \left(k \omega_{0} t+\varphi_{k}\right)
\end{aligned}
$$

where $\phi=\omega_{0} \Delta t$ is the applied phase shift. Each matched generator in Fig. 1 can be clocked to generate time-shifted versions of the same sinusoidal signal with different delays $\Delta t_{i}$. Let us now define signal $y(t)$ as the sum,

$$
y(t)=x_{1}(t)+\sum_{i=2}^{(n-1) / 2}\left[x_{1}\left(t+\Delta t_{i}\right)+x_{1}\left(t-\Delta t_{i}\right)\right]
$$

where $n-1$ matched generators are used to provide signals of opposite delays, $\Delta t_{i}$ and $-\Delta t_{i}$, respect to signal $x_{1}(t)$. From equations (2), (3), (4) it can be derived,

$$
y(t)=\sum_{k=1}^{\infty} A_{k}\left[1+2 \sum_{i=2}^{(n-1) / 2} \cos \left(k \phi_{i}\right)\right] \cos \left(k \omega_{0} t+\varphi_{k}\right)
$$


where $\phi_{i}=\omega_{0} \Delta t_{i}$. That is, as a result of combining timeshifted versions of the original signal $x_{1}(t)$ with opposite timeshifts, we can build a signal $y(t)$ whose spectral content is that of signal $x_{1}(t)$ scaled by coefficient $1+2 \sum_{i=2}^{(n-1) / 2} \cos \left(k \phi_{i}\right)$. If phase-shifts $\phi_{i}$ are properly selected such that these coefficients are small, unwanted harmonic components in $y(t)$ can be attenuated or completely cancelled.

\section{Practical Implementation}

A direct implementation of the generator based on the conceptual block diagram in Fig. 1 is clearly not practical. The use of $n$ matched generators is costly in terms of power and silicon area. Additionally, any mismatch between the generated signals would have a negative impact in the harmonic cancellation. Instead of that, we propose to perform the harmonic cancellation (4) at the input of a single basic generator by taking advantage of its programmable input levels.

Let us consider the step-wise sine-wave defined by the programable input elements of the basic generator in Fig. 2,

$$
f(t)=\sin \left(k \frac{2 \pi}{N}\right) \quad \frac{k T}{N} \leq t<\frac{(k+1) T}{N}
$$

where $N$ is the number of steps in one period of the stepwise sinusoid, $T$ is its period, and amplitude values have been normalized for simplicity. Let us define $f_{+m}(t)$ and $f_{-m}(t)$ as time-shifted versions of $f(t)$,

$$
f_{ \pm m}(t)=\sin \left((k \pm m) \frac{2 \pi}{N}\right) \quad \frac{k T}{N} \leq t<\frac{(k+1) T}{N}
$$

where $m$ is an integer number, $1 \leq m \leq N / 2$. By restricting time-delays to this set of integer shifts, functions $f(t), f_{+m}(t)$ and $f_{-m}(t)$ can be built with the same set of sine-wave samples. That is, these functions can be generated with the same set of programmable input elements at the generator input.

In order to perform the harmonic cancellation, we propose to modify the programmable input elements in the basic generator to perform the sum,

$$
f_{y}(t)=f(t)+\sum_{m}\left[f_{-m}(t)+f_{+m}(t)\right]
$$

which is analogous to (4). Function $f_{y}(t)$ is also a periodic step-wise function with $N$ steps per period. If we assume $n$ different $m$ values, then each step in $f_{y}(t)$ is the sum of $2 n+1$ terms, and according to (6)-(7) all these terms correspond to the same set of sampled values of the original $N$-step sine-wave (6). This summation can be then performed at the programmable input of the generator by connecting to the signal path the $2 n+1$ elements needed for each step in (8) simultaneously. The only modifications with respect to the original generator are then the necessary control signals controlling the programmable elements and, depending on the values of $n, m$, and $N$, some of the original elements may have to be replicated.

In order to illustrate the proposed implementation strategy, Fig. 3 shows the schematic of a switched-capacitor implementation of the proposed sinusoidal generator including harmonic
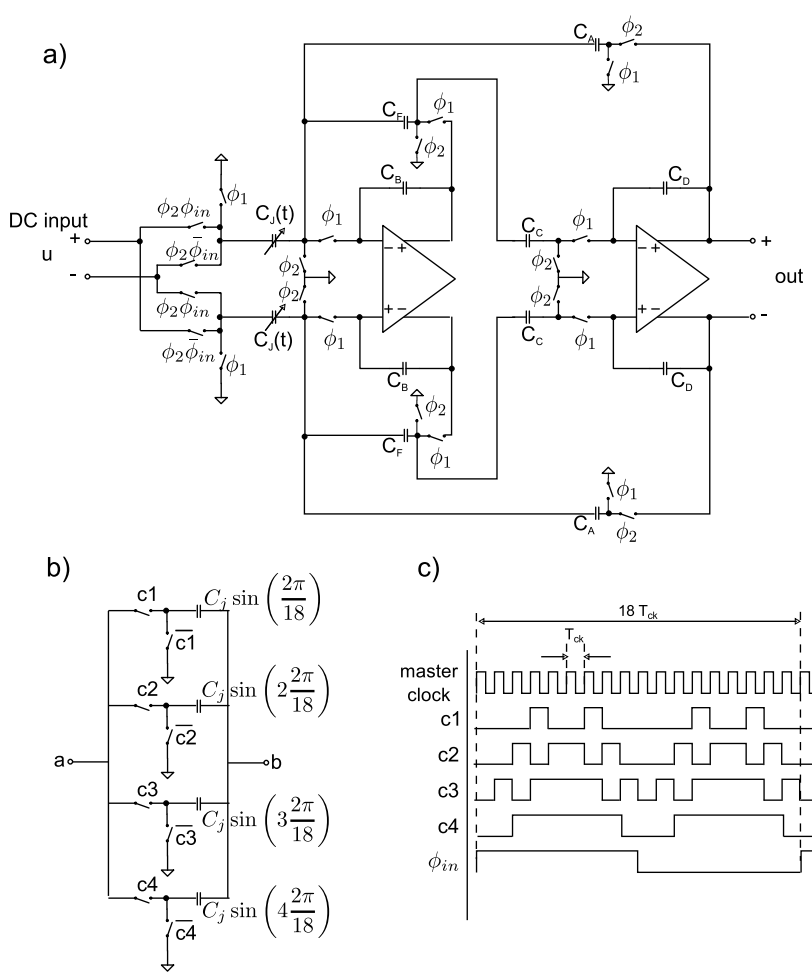

c)

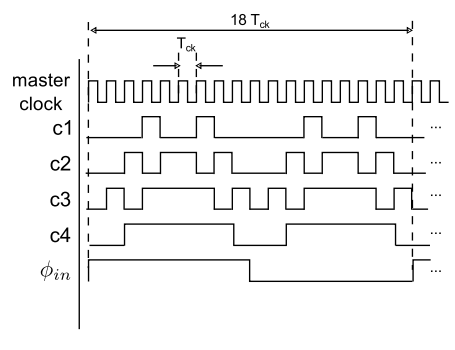

Fig. 3. a) Practical SC implementation of the proposed generation technique; b) Programmable input capacitor; c) Master clock and control signals for three phase-shifted sinusoids of phases $0,+2 \pi / 9,-2 \pi / 9$.

cancellation. This generator performs at the programmable input the sum of three 18-step sinusoids, with phases 0 , $+2 \pi / 9$, and $-2 \pi / 9$ (i.e. $N=18, m=2$, and $n=1$ ). This choice results in the cancellation of the third harmonic component. Also notice that $N, m$, and $n$ have been chosen to avoid replicating any input elements: the input array is the same one needed for building a single 18-step sinusoid. Only the control signals of the programmable input have been modified respect to the basic generator.

This generator design has been derived from a fullydifferential Fleischer-Laker biquad [9] in a lowpass configuration. The system operates with two non-overlapped clock phases $\phi_{1}$ and $\phi_{2}$. The corner frequency of the filter has been placed at one-eighteenth of the clock frequency to make it coincident with the fundamental frequency of the generated signal and to provide attenuation for higher harmonic components. This way, harmonic cancellation and analog filtering of the generated signal are combined on a single block.

\section{Simulation Results}

The sinusoidal signal generator with harmonic cancellation presented in Fig. 3 was modeled according to the guidelines in [2]. The developed behavioral model takes into account the number of steps per period in the generated signal: $N=18$, the number of generated phases: $2 n+1=3$, the selected delay: $m=2$, and possible random errors affecting each programmable input element due to mismatch, process 


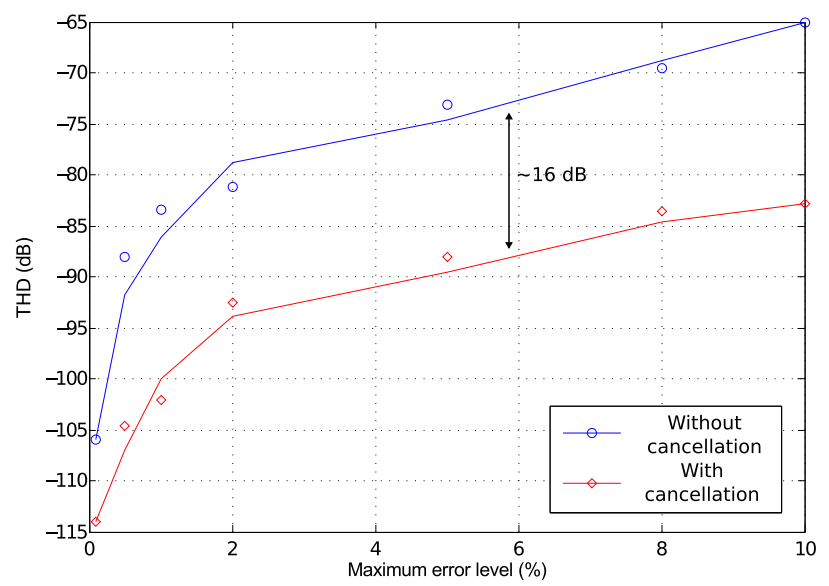

Fig. 4. THD of the generated sinusoid vs. error level in the input elements, with and without harmonic cancellation.

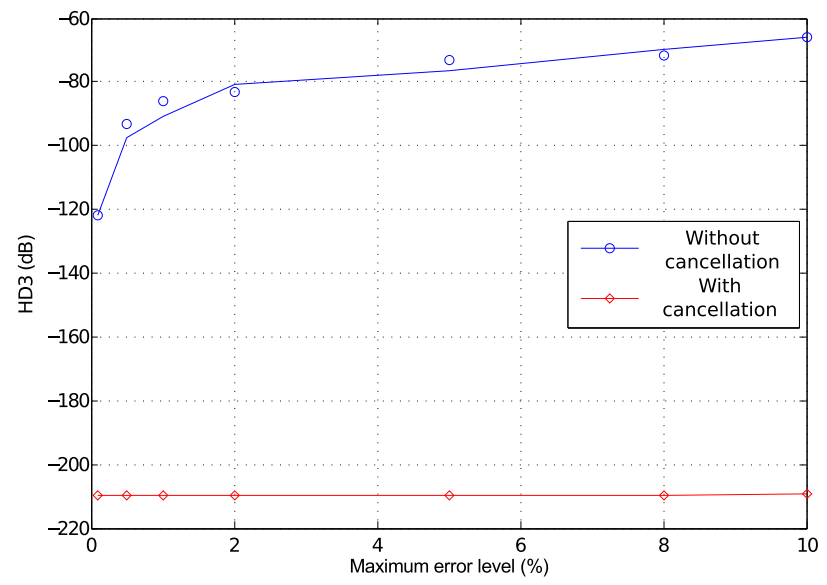

Fig. 5. Third order harmonic distortion of the generated sinusoid vs. error level in the input elements, with and without harmonic cancellation.

variations, charge injection, etc. The developed model was used to estimate the performance of the generator in terms of THD by statistical simulations. For comparison purposes we considered also the corresponding generator without harmonic cancellation (the control signals of the programmable input are modified for the latter case to generate only one of the sinusoidal signals, as in [2]).

Fig. 4 shows the obtained THD for the generated sinusoid signals at the output of the generator as a function of the error level of the input elements, with and without harmonic cancellation. THD figures are greatly improved due to the application of the harmonic cancellation technique. As it can be seen in Fig. 4, THD is boosted around $16 \mathrm{~dB}$ for error levels above $2 \%$. Fig. 5 , on the other hand, shows the third-order harmonic distortion as a function of the error level of the input elements, again with and without harmonic cancellation. It is clear to see that the cancellation strategy effectively attenuates the third harmonic component independently of the error levels of the input elements.

Obtained results suggest that the application of the harmonic cancelation technique not only improves the linearity of the original generator, but it also makes it less sensitive to any random errors in the input elements. This is an advantage compared to previously presented generators using harmonic cancelation such as [6], [8]. These previous works are based on matching different stages for the generation of time-delayed signals, so any mismatch between the stages has negative impact on the harmonic cancellation. In our proposed generator the three time-delayed signals used for harmonic cancellation are built simultaneously from the same elements, reducing this way the sensitivity to mismatch.

\section{CONCLUSIONS}

We have presented a methodology for the generation of analog sinusoidal signals using a harmonic cancellation technique. The proposed generator is based on a modified low-order ana$\log$ filter with a DC input and programmable input elements, in such a way that the filter performs signal generation, harmonic cancellation and filtering of the unwanted components.

Simulation results show a significant improvement in the linearity of the generated signal compared to the generator without harmonic cancellation and a lower sensitivity to mismatch compared to previous generators with harmonic cancellation.

\section{ACKNOWLEDGMENT}

This work has been partially funded by a CSIC JAEDoc contract (cofinanced by FSE), the Junta de Andalucia project P09-TIC-5386, and the Ministerio de Economia y Competitividad project TEC2011-28302, both of them cofinanced by the FEDER program.

\section{REFERENCES}

[1] F. Poehl, F. Demmerle, J. Alt, and H. Obermeir, "Production test challenges for highly integrated mobile phone SOCs: A case study," in 15th IEEE European Test Symposium (ETS), 24-28 May 2010, pp. 17-22.

[2] M. Barragán, D. Vázquez, and A. Rueda, "Analog sinewave signal generators for mixed-signal built-in test applications," Journal of Electronic Testing, vol. 27, no. 3, pp. 305-320, 2011.

[3] B. Dufort and G. W. Roberts, "On-chip analog signal generation for mixed-signal built-in self-test," Solid-State Circuits, IEEE Journal of, vol. 34, no. 3, pp. 318-330, Mar 1999.

[4] G. Prenat, S. Mir, D. Vázquez, and L. Rolíndez, "A low-cost digital frequency testing approach for mixed-signal devices using modulation," Microelectronics Journal, vol. 36, no. 12, pp. 1080-1090, 122005.

[5] M. A. Domínguez, J. L. Ausín, J. F. Duque-Carrillo, and G. Torelli, "A 1mhz area-efficient on-chip spectrum analyzer for analog testing," Journal of Electronic Testing, vol. 22, no. 4-6, pp. 437-448, 2006.

[6] F. Bahmani and E. Sanchez-Sinencio, "Low thd bandpass-based oscillator using multilevel hard limiter," Circuits, Devices \& Systems, IET, vol. 1, no. 2, pp. 151-160, April 2007.

[7] M. M. Elsayed and E. Sanchez-Sinencio, "A low thd, low power, high output-swing time-mode-based tunable oscillator via digital harmoniccancellation technique," Solid-State Circuits, IEEE Journal of, vol. 45, no. 5, pp. 1061-1071, May 2010.

[8] B. K. Vasan, S. K. Sudani, D. J. Chen, and R. L. Geiger, "Sinusoidal signal generation for production testing and bist applications," Circuits and Systems (ISCAS), 2012 IEEE International Symposium on, pp. 26012604, 20-23 May 2012.

[9] K. R. Laker and S. W. M. C., Design of analog integrated circuits and systems. McGraw-Hill International Edition, 1994. 